\title{
BeppoSAX/PDS identification of the true counterpart of the Piccinotti source H0917-074
}

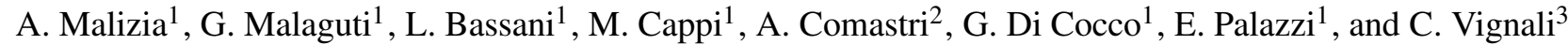 \\ ${ }^{1}$ IASF/CNR, via Piero Gobetti 101, 40129 Bologna, Italy \\ 2 INAF/Osservatorio Astronomico di Bologna, via Ranzani 1, 40127 Bologna, Italy \\ 3 Department of Astronomy and Astrophysics, Pennsylvania State University, 525 Davey Lab, University Park, PA 16802, USA
}

Received 20 June 2002 / Accepted 5 August 2002

\begin{abstract}
High energy emission has been discovered serendipitously by the BeppoSAX/PDS telescope in the $\sim 1.3^{\circ}$ field of view around the Piccinotti source H0917-074. A re-pointing of BeppoSAX/NFI has allowed the association of this emission with the Seyfert 2 galaxy MCG-1-24-12 which lies within the original HEAO1/A2 error box of H0917-074. This is the first PDS serendipitous discovery of a Seyfert 2 galaxy and the first detection of MCG-1-24-12 in the X-ray domain. The measured 2-10 keV flux of MCG-1-24-12 is $\sim 1 \times 10^{-11} \mathrm{erg} \mathrm{cm}^{-2} \mathrm{~s}^{-1}$ compatible with the Piccinotti HEAO-1/A2 observation. This is a factor of $\sim 6$ greater than that observed from EXO0917.3-0722, originally suggested as the counterpart of the Piccinotti source. The 2-10 keV spectrum of MCG-1-24-12 shows the presence of Fe $\mathrm{K}_{\alpha}$ emission together with an absorption feature at $\sim 8.7 \mathrm{keV}$. At high energies, the Seyfert 2 still dominates and the observed $20-100 \mathrm{keV}$ flux is $\sim 4 \times 10^{-11} \mathrm{erg} \mathrm{cm}^{-2} \mathrm{~s}^{-1}$.
\end{abstract}

Key words. X-rays: galaxies - galaxies: Seyfert - galaxies: individual: MCG-1-24-12

\section{Introduction}

The Piccinotti sample (Piccinotti et al. 1982) is to date the only statistically complete sample of active galactic nuclei (AGN) in the $2-10 \mathrm{keV}$ energy range. It was obtained using data from the A2 experiment on the HEAO-1 satellite which performed a survey of $8.3 \mathrm{sr}$ of the sky (65.5\% coverage) with $|b| \geq 20^{\circ}$, at a limiting flux of $3.1 \times 10^{-11} \mathrm{erg} \mathrm{cm}^{-2} \mathrm{~s}^{-1}$. The sample was composed of 36 AGN: 30 Seyfert galaxies, one starburst galaxy (M82), 4 BL Lac objects and one QSO (3C273).

The BATSE instrument on board CGRO provided, for the first time, a systematic coverage of the whole sample at higher energies (20-100 keV range, Malizia et al. 1999) and for the brightest sources OSSE observations in the $50-500 \mathrm{keV}$ band have also been performed (McNaron-Brown et al. 1995; Zdziarski et al. 2000). BeppoSAX has observed almost the whole Piccinotti sample in the broad band $0.1-200 \mathrm{keV}$ energy range, with the exception of the three Seyfert galaxies: IIIZW2, MKN 590 and NGC 3227.

We have carried out a multiyear project to observe with BeppoSAX-NFI the poorly studied (i.e. fainter) sources of the Piccinotti sample among which is the galaxy H0917074 which was identified with the QSO EXO0917.3-0722. Thanks to the BeppoSAX-PDS observation we discovered that EXO0917.3-0722 is contaminated by another hard X-ray source subsequently identified with the Seyfert 2

Send offprint requests to: A. Malizia, e-mail: malizia@bo.iasf.cnr.it galaxy MCG-1-24-12, which is the true X-ray emitter detected by the A2 instrument.

It is worth noting that the high sensitivity of the PDS instrument (Frontera et al. 1997) on board the BeppoSAX satellite has provided the opportunity to increase the number of Seyfert 2 s detected up to $100 \mathrm{keV}$, improving our understanding of the high energy characteristics of this type of object. BeppoSAX observations of Seyfert 2 galaxies have demonstrated that these objects can be powerful hard X-ray emitters (above $10 \mathrm{keV}$ ) even though their $2-10 \mathrm{keV}$ radiation is severely attenuated by absorption in thick material (Bassani et al. 1999). In fact, hard X-ray spectra are probably the best tool to directly measure the absorption affecting Seyfert 2 nuclei.

In this paper the BeppoSAX broad band spectrum of the first PDS serendipitous discovery of a Seyfert 2 galaxy, MCG-1-24-12, is presented. From this study, which is also the first of this source in the X-ray domain, MCG-1-24-12 turns out to be a Compton thin Seyfert 2 galaxy $\left(N_{\mathrm{H}} \sim 7 \times\right.$ $10^{22}$ atoms $\mathrm{cm}^{-2}$ ) with a slightly peculiar spectrum.

\section{Observations: From EXO0917.3-0722 to MCG-1-24-12}

Within the multiyear project of completing the observation of poorly studied Piccinotti sample sources, H0917-074 was targeted by the BeppoSAX NFI from April 15th to April 17th, 2000. This source was unidentified in the original Piccinotti list and has been subsequently associated to 


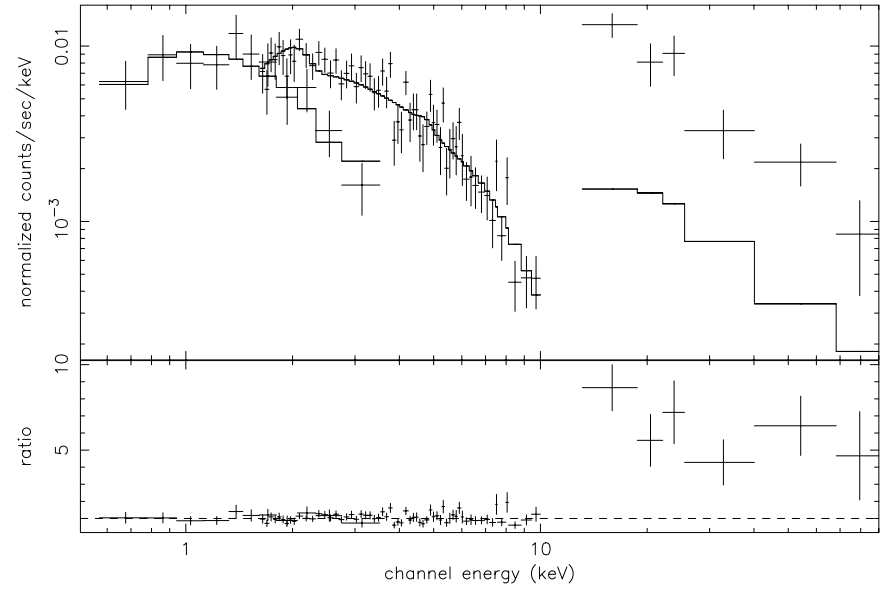

Fig. 1. The LECS MECS and PDS spectrum of H0917-074 (April 2000 pointing) fitted with a simple power law model of $\Gamma=$ 1.76.

the QSO/Seyfert 1 galaxy EXO0917.3-0722 $(z=0.169)$ by Giommi et al. (1991) using EXOSAT observations. More recently, the source has been observed by ROSAT in soft Xrays (Schartel et al. 1997) and by BATSE on-board CGRO in the hard X-ray band (Malizia et al. 1999). A quick-look inspection of the BeppoSAX MECS-PDS broad band spectrum of EXO0917.3-0722 indicates an apparent inconsistency. In fact, as shown in Fig. 1, a simple power-law fit requires a MECS-PDS normalization constant of $\sim 8-10$, i.e. an order of magnitude greater than that normally used (Fiore et al. 1998). The simplest explanation for this phenomenon is the presence of a contaminating serendipitous source in the field of view of the PDS, which is hexagonal in shape and 78 arcmin wide.

The region of the sky around EXO0917.3-0722 was searched for possible high energy sources, and the only possible candidate found was the Seyfert $2 \mathrm{MCG}-1-24-12(z=$ 0.0198), which also lies within the original HEAO 1/A2 error box (Piccinotti et al. 1982). In Fig. 2 the original A2 error box for H0917-074 is shown with the position of MCG-1-24-12 superimposed. MCG-1-24-12 has an optical magnitude of $m_{v}=15$ and it is in the 2MASS catalogue $\left(m_{J}=14.2\right.$, $m_{H}=13.3, m_{K}=12.8$ ); it belongs to the $60 \mu \mathrm{m}$ sample of warm IRAS galaxies (de Grijp et al. 1992; Kinney et al. 2000) with a $\log \left(L_{\mathrm{FIR}}\right)=10.01 L_{\odot}$ (Chatzichristou 2000). It is also a radio galaxy with a $F_{3.6 \mathrm{~cm}}=9.1 \mathrm{mJy}$ and its $3.6 \mathrm{~cm}$ radio contours show a $\sim 70$ pc elongation towards the west (Schmitt et al. 2001), but it was never observed in the X-ray band. After finding MCG-1-24-12 as the possible candidate, we were able to obtain a pointed observation with BeppoSAX and found that its $2-10 \mathrm{keV}$ flux of $\sim 1 \times 10^{-11} \mathrm{erg} \mathrm{cm}^{-2} \mathrm{~s}^{-1}$ is more consistent with that reported in the original Piccinotti source list (Piccinotti et al. 1982) than that observed from EXO0917.3-0722. This result indicates that the Seyfert 2 is the correct identification of H0917-074. Also BATSE detected from this region a 20$100 \mathrm{keV}$ flux of $\sim 5 \times 10^{-11} \mathrm{erg} \mathrm{cm}^{-2} \mathrm{~s}^{-1}$ (Malizia et al. 1999) which is in perfect agreement with the PDS 20-100 keV flux of $\sim 4 \times 10^{-11} \mathrm{erg} \mathrm{cm}^{-2} \mathrm{~s}^{-1}$ reported here from MCG-1-24-12, suggesting that it is this source that was observed.

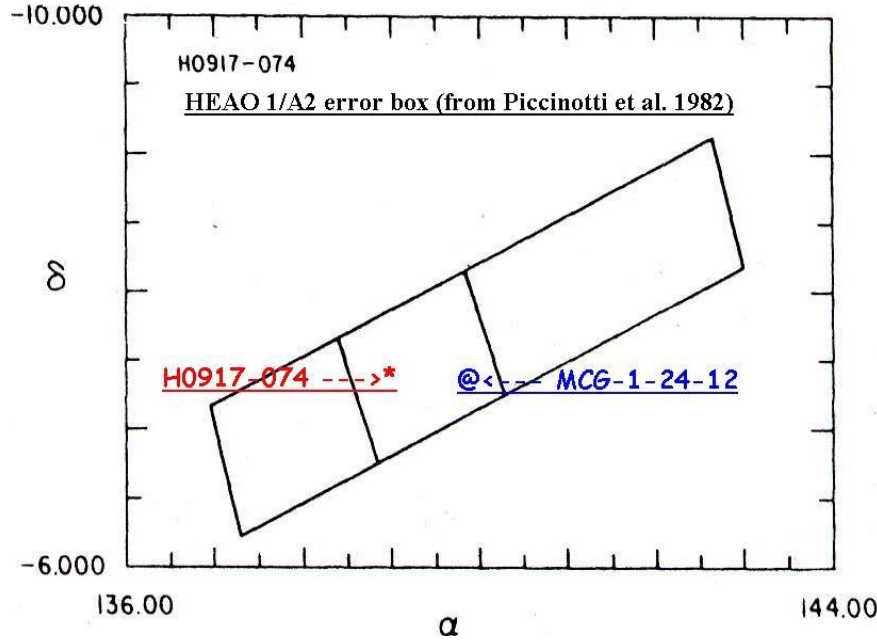

Fig. 2. The HEAO-1/A2 error box for H0917-074 adapted from Piccinotti et al. (1982). $\alpha$ and $\delta$ are in degrees (equinox 1950). The inner and outer boxes are the $90 \%$ confidence boxes as described in Marshall et al. (1979). The BeppoSAX coordinates for H0917-074 and MCG-1-24-12 are superimposed.

\section{Spectral analysis}

Standard data reduction was performed using the software package "SAXDAS" (see http://www.sdc.asi.it/software and the Cookbook for BeppoSAX NFI spectral analysis, Fiore et al. 1998). Data were linearized and cleaned for Earth occultation periods and unwanted periods of high particle background (satellite passages through the South Atlantic Anomaly). The LECS, MECS and PDS backgrounds are relatively low and stable (variations of at most $30 \%$ during the orbit) thanks to the satellite's low inclination orbit (3.95 degrees). Data were accumulated for Earth elevation angles $>5$ degrees and magnetic cut-off rigidity $>6$. For the PDS data we adopted a fine energy and temperature dependent Rise Time selection, which decreases the PDS background by $\sim 40 \%$, improving the signal to noise ratio of faint sources by about 1.5 (Frontera et al. 1997; Fiore et al. 1998). Spectral fits were performed using the XSPEC 11.0.1 software package and public response matrices as from the 1998 November issue. PI (Pulse Invariant) channels are rebinned in such a way as to sample the instrument resolution with the same number of channels at all energies when possible and to have at least 20 counts per bin. This allows the use of the $\chi^{2}$ method in determining the best fit parameters, since the distribution in each channel can be considered Gaussian. Constant factors have been introduced in the fitting models in order to take into account the intercalibration systematic uncertainties between instruments (Fiore et al. 1998). All the quoted errors correspond to $90 \%$ confidence level for two interesting parameters. The models used in what follows contain an additional term to allow for the absorption of X-rays due to our galaxy, which in the direction of both EXO0917.3-0722 and MCG-1-24-12 corresponds to $N_{\mathrm{H}}=3.5 \times 10^{20}$ atoms $\mathrm{cm}^{-2}$. 


\subsection{EXO0917.3-0722}

BeppoSAX pointed at EXO0917.3-0722 for an effective exposure time of $10 \mathrm{ks}$ for the LECS, $52 \mathrm{ks}$ for the MECS, and $25 \mathrm{ks}$ for the PDS. Spectral data were extracted from a region centred on EXO0917.3-0722 with a radius of 4 arcmin. The net count rate was $1.67 \pm 0.14 \times 10^{-2} \mathrm{cts} / \mathrm{s}$ in the LECS, $2.48 \pm 0.07 \times 10^{-2} \mathrm{cts} / \mathrm{s}$ in the MECS, and $0.28 \pm 0.03 \mathrm{cts} / \mathrm{s}$ in the PDS. LECS (0.1-4 keV) and MECS (2-10 keV) data are well fitted by a simple power law of photon index $\Gamma=1.76 \pm 0.09$ absorbed by the Galactic column density $\left(\chi^{2} / v=90.7 / 91\right)$. The addition of an iron line is not required by the data. If a narrow line $(\sigma=0)$ is fixed at $6.4 \mathrm{keV}$ energy, the upper limit of its equivalent width is $E W<210 \mathrm{eV}$. The $0.1-10 \mathrm{keV}$ spectrum of EXO0917.3-0722 is consistent with the average spectral characteristics of a sample of 10 low redshift quasars observed by BeppoSAX (Mineo et al. 2000). The $2-10 \mathrm{keV}$ flux is $1.7 \times 10^{-12} \mathrm{erg} \mathrm{cm}^{-2} \mathrm{~s}^{-1}\left(L_{\mathrm{X}} \sim 9 \times 10^{43} \mathrm{erg} \mathrm{s}^{-1}\right)$ which is roughly an order of magnitude less than the A2 measurement of $3.4 \times 10^{-11} \mathrm{erg} \mathrm{cm}^{-2} \mathrm{~s}^{-1}$ (Piccinotti et al. 1982).

\subsection{MCG-1-24-12}

The BeppoSAX-NFIs pointed at MCG-1-24-12 from May 15th to May 17th, 2001. The effective exposure times are $28 \mathrm{ks}$ for the LECS, $76 \mathrm{ks}$ for the MECS, and $36 \mathrm{ks}$ for the PDS. Spectral data were extracted from a region centred on MCG-1 - 24-12 with a radius of 4 arcmin. LECS and MECS background spectra were extracted from blank sky fields using regions of the same size in detector coordinates. The net count rate was $8.5 \pm 0.6 \times 10^{-3} \mathrm{cts} / \mathrm{s}$ in the (1-4 keV) LECS energy band, $9.1 \pm 0.1 \times 10^{-2} \mathrm{cts} / \mathrm{s}$ in the $(2-10 \mathrm{keV}) \mathrm{MECS}$ energy range, and $0.43 \pm 0.03 \mathrm{cts} / \mathrm{s}$ in the $(20-100 \mathrm{keV})$ PDS band. Source plus background light curves did not show any significant variation, therefore timing analysis has not been performed and all data have been grouped for spectral analysis. The main results are summarized in Table 1.

Due to the low statistical quality of the data at lower energies, only data above $1 \mathrm{keV}$ have been considered. The broad band (1-100 keV) spectrum of MCG-1-24-12 was firstly modeled with a flat, absorbed power law plus a Gaussian line (model 1, Table 1). The results are $\Gamma=1.59_{-0.03}^{+0.07}$ and $N_{\mathrm{H}}=$ $6.27_{-0.57}^{+0.53} \times 10^{22} \mathrm{~cm}^{-2}$ while the line is centered at $6.4_{-0.18}^{+0.16} \mathrm{keV}$ with the equivalent width of $E W=176_{-61}^{+61} \mathrm{eV}$. The line is consistent with being narrow at the $99 \%$ confidence level, therefore we have fixed the value of its width (sigma) to zero. The residuals clearly show evidence of two more features: an absorption edge at $E \sim 8 \mathrm{keV}$ and a possible reflection hump at around $30 \mathrm{keV}$. We have therefore considered an absorption edge to account for the deficit at around $8 \mathrm{keV}$. This turns out to be located at $E=8.4_{-0.6}^{+0.4} \mathrm{keV}$, with optical depth $\tau=0.26_{-0.16}^{+0.17}$. The addition of this absorbed feature to the previous model is statistically significant at $>98 \%$ confidence using the F-test $\left(\chi^{2}\right.$ decreases by 12 for 2 more fitting parameters). The presence of the feature has been confirmed as it is still detected when using different background event files extracted from blank areas in the field of view of MCG-1-24-12. Even taking into account the presence of the absorption edge, the data to model

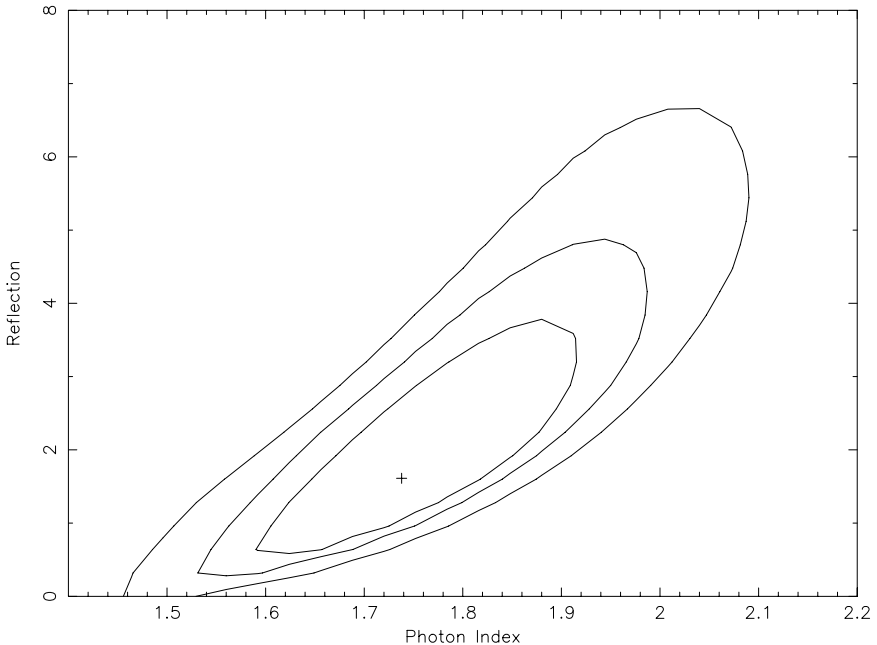

Fig. 3. 68, 95 and 99 per cent confidence contours of reflection versus photon index for model 3.

ratio still shows residuals at around $8 \mathrm{keV}$ which cannot be fitted by adding one more edge. Therefore we have replaced the edge with a notch line model which is equivalent to a very saturated absorption line (model 2). The quality of the fit improves: $\Delta \chi^{2}=6$ with the same degrees of freedom. The energy of the absorption line is now $8.7_{-0.15}^{+0.4} \mathrm{keV}$ and the line width is $0.23_{-0.11}^{+0.11} \mathrm{keV}$ (see 3.2.1).

For the excess at $30 \mathrm{keV}$, we take into account a possible reflection component (model 3). When introduced, the reflection turns out to be strong $\left(R=1.62_{-1.12}^{+3.38}\right)$ with a cut-off at $E_{\mathrm{c}}>80 \mathrm{keV}$. The photon index is compatible with the expected canonical value $\Gamma=1.74_{-0.27}^{+0.36}$ of Seyfert 2 galaxies. In Fig. 3 the confidence contours of the reflection component versus the photon index are shown. The energies of both the emission line and the absorption line do not change significantly from the previous ones. In Fig. 4, the confidence contours of the notch are shown for this more complex model. The addition of the reflection component further improves the quality of the fit providing us with our best-fit model. In Fig. 5 the $1-100 \mathrm{keV}$ broad band spectrum of MCG-1-24-12 fitted with model 3 is shown.

\subsubsection{The absorption feature at $\sim 8 \mathrm{keV}$}

The best fit model that explains all the features of the $1-100 \mathrm{keV}$ spectrum of MCG-1-24-12 is an absorbed power law plus a Compton reflection component $(R \sim 1.6)$ from a cold slab isotropically illuminated by the power law photons as in the PEXRAV model in XSPEC by Magdziarz \& Zdziarski (1995), an Iron $\mathrm{K} \alpha$ line at energy $E_{\text {line }} \sim 6.4 \mathrm{keV}$ and an absorption feature at energy $E_{\text {notch }} \sim 8.7 \mathrm{keV}$.

The broad band spectrum of MCG-1-24-12 is consistent with that generally found in Seyfert 2 objects, but the detection of the absorption line makes the spectrum of MCG-1-24-12 rather peculiar. Generally, the detection of an absorption edge indicates the presence of a warm photoionized absorber along the line of sight; therefore we have tried to add a uniform warm absorber in this model as described by the ABSORI model 
Table 1. MCG-1-24-12: spectral analysis.

\begin{tabular}{clllllllll}
\hline \hline Model & $N_{\mathrm{H}} \times 10^{22}$ & $\Gamma$ & $E_{\text {Line }}(\mathrm{keV})$ & $E W(\mathrm{eV})$ & $E_{\text {Notch }}(\mathrm{keV})$ & Width $_{\text {Notch }}(\mathrm{keV})$ & $R$ & $E_{\mathrm{c}}(\mathrm{keV})$ & $\chi^{2} / v$ \\
\hline$(1)$ & $6.27_{-0.57}^{+0.53}$ & $1.59_{-0.03}^{+0.07}$ & $6.40_{-0.18}^{+0.16}$ & $176_{-61}^{+61}$ & - & - & - & & $95 / 62$ \\
$(2)$ & $6.25_{-0.45}^{+0.45}$ & $1.57_{-0.07}^{+0.07}$ & $6.40_{-0.20}^{+0.15}$ & $157_{-61}^{+58}$ & $8.70_{-0.15}^{+0.40}$ & $0.23_{-0.11}^{+0.11}$ & - & - & $77 / 60$ \\
$(3)$ & $6.54_{-0.61}^{+0.63}$ & $1.74_{-0.27}^{+0.36}$ & $6.38_{-0.18}^{+0.20}$ & $130_{-55}^{+61}$ & $8.72_{-0.19}^{+0.15}$ & $0.20_{-0.12}^{+0.07}$ & $1.62_{-1.12}^{+3.38}$ & $>80$ & $68 / 58$ \\
\hline
\end{tabular}

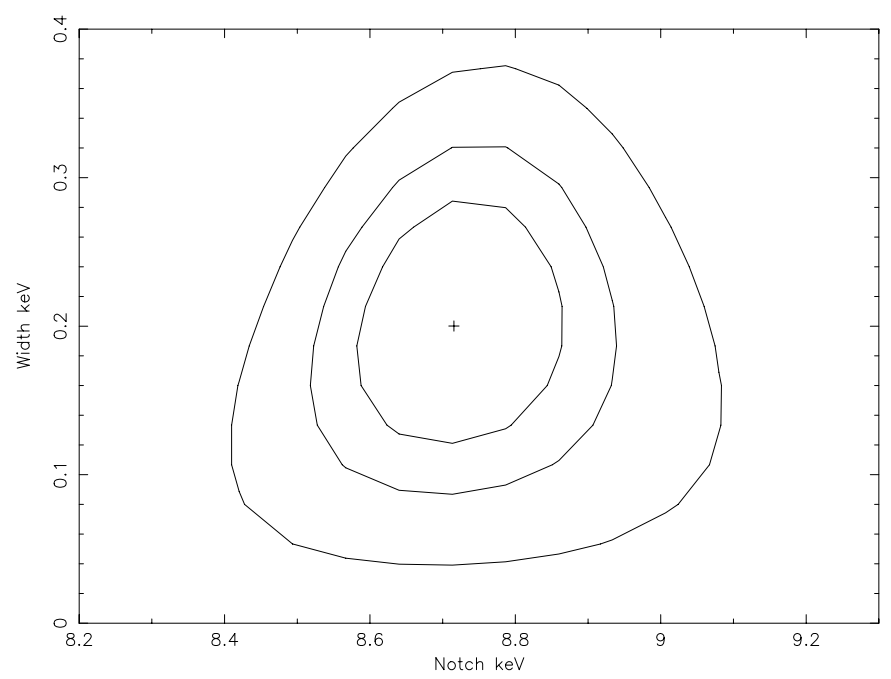

Fig. 4. 68, 95 and 99 per cent confidence contours of the notch: width versus energy of the notch.

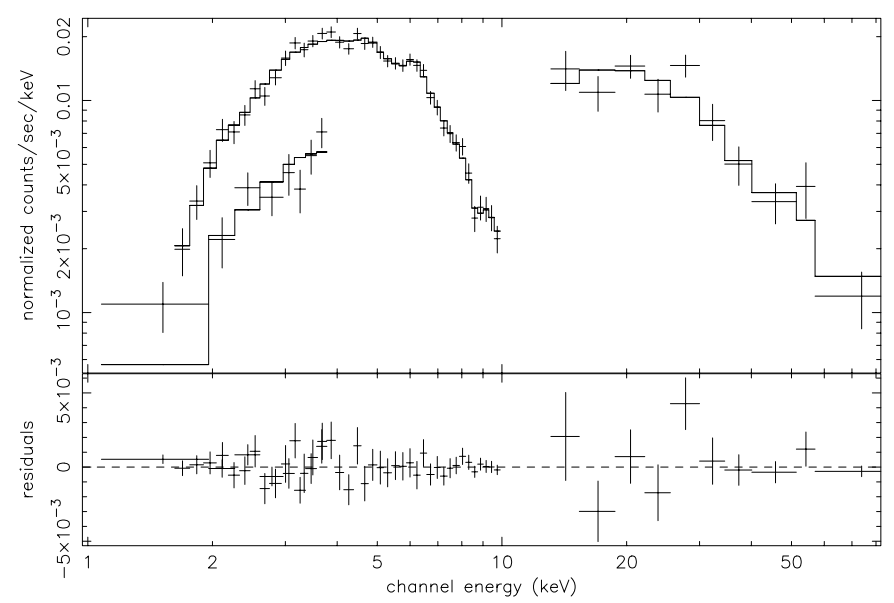

Fig. 5. The LECS MECS and PDS spectrum of MCG-1-24-12 fitted with model 3 (best-fit model) in Table 1.

(Done et al. 1992). The energy of the edge ( $\sim 8 \mathrm{keV})$ strongly suggests that the absorption is mainly due to FeXXIII-FeXXV. Assuming Dalgarno \& Layzer (1987) abundances, the photoelectric cross section of neutral Fe per hydrogen atom is about $7 \times 10^{-25}$; therefore, taking the optical depth to be $\sim 0.2$, as observed, the column density of the warm material $\left(N_{\mathrm{H}_{\mathrm{W}}} \sim \tau / \sigma\right)$ is found to be $\sim 3 \times 10^{23}$ atoms $\mathrm{cm}^{-2}$.

Fixing to this value the $N_{\mathrm{H}_{\mathrm{W}}}$ parameter in the ABSORI model, the fit gives an ionization parameter $X_{i} \sim 290$ that is still compatible with the the presence of a neutral Fe line $\left(E_{\text {line }} \sim 6.4 \mathrm{keV}\right)$ but some residuals at $\sim 8 \mathrm{keV}$ still remain and the quality of the fit is worst $\left(\chi^{2} / v=130 / 57\right)$.
Another way to explain the production of the absorption edge in the spectrum of MCG-1-24-12 is by reflection from a ionized disk (PEXRIV model) which has recently been used successfully to fit the BeppoSAX data of a sample of Seyfert 1 objects (De Rosa et al. 2001). As generally observed for the Seyfert $1 \mathrm{~s}$, the spectrum turns out to be flat $(\Gamma \sim 1.64)$, the reflection component is $R \sim 0.4$, and the ionization parameter is around 200; however, again the quality of the fit does not improve $\left(\chi^{2} / v=81 / 55\right)$ with respect to the neutral reflection model. We conclude that neither of these two models which allow for the presence of warm material at the source, provide a better fit than simple reflection from a standard cold disk. This may be due to the fact that both the ABSORI and PEXRIV models are more appropriate to fit edge-like features and not an absorption line such as that found in our data.

An absorption feature at around $8 \mathrm{keV}$ has been detected in a few Seyfert galaxies, e.g. in M81 (Pellegrini et al. 2000) and in NGC 3516 (Nandra et al. 1999; Constantini et al. 2000) which has been explained recently in terms of the influence of resonant absorption on the iron emission line by Ruszkowski \& Fabian (2000). However, it is worth noting that the BeppoSAX/MECS instrument has not sufficient resolution at these energies to resolve the line. With our data we can conclude that the absorption feature is produced by ions from FeXXIII to FeXXV (8.5-8.9 keV) within the $90 \%$ confidence interval, while its best fit energy $(8.7 \mathrm{keV})$ corresponds to the FeXXIV K-edge (Makishima 1985). These values are marginally compatible with the energy of the line at $6.38_{-0.18}^{+0.20} \mathrm{keV}$. Also the $E W$ of the emission line and the width of the absorption line are consistent within the uncertainties of the various parameters with the measured column density of $N_{\mathrm{H}}=6.5_{-0.61}^{+0.63} \times 10^{22} \mathrm{~cm}^{-2}$.

Higher quality data such as those possible with XMMNewton will provide definite evidence for this feature and will help to better investigate its nature.

\section{Conclusions}

The main result of this work is that the X-ray source corresponding to H0917-074 in the only 2-10 keV complete sample of AGN (Piccinotti et al. 1982) is the Seyfert 2 MCG-1-24-12 instead of the QSO/Seyfert 1 EXO0917.3-0722. MCG-1-2412 was never before observed in the X-ray domain and from the present study it turns out to be a Compton thin Seyfert 2 galaxy $\left(N_{H} \sim 7 \times 10^{22}\right.$ atoms $\left.\mathrm{cm}^{-2}\right)$ with an almost standard spectrum. The only peculiarity in this spectrum is an absorption feature at around $8 \mathrm{keV}$ which cannot be explained by the presence of warm material around the source. Our best fit model is a power law absorbed by uniform cold material and reflected from a standard cold disk. 
With this observation the number of Seyfert 2 s belonging to the Piccinotti sample grows to 8 out of 30 Seyfert galaxies: all these objects have column densities $N_{\mathrm{H}}>10^{22}$ atoms $\mathrm{cm}^{-2}$. In addition a few type 1 Seyferts in the Piccinotti sample have $N_{\mathrm{H}}$ exceeding this value (NGC 4151, Zdziarski et al. 2002; NGC 526A, Landi et al. 2001; NGC 3783, De Rosa et al. 2002). This sample is complete down to a flux limit of $3.1 \times 10^{-11} \mathrm{erg} \mathrm{cm}^{-2} \mathrm{~s}^{-1}$ and therefore can be used to estimate with some confidence the percentage of absorbed sources at these high fluxes: out of 31 objects identified with Seyfert/QSO (Malizia et al. 1999 and this paper), 11 are absorbed above $10^{22}$ atoms $\mathrm{cm}^{-2}$, implying that a consistent fraction $(30 \pm 12 \%)$ of all AGN in the $2-10 \mathrm{keV}$ band are absorbed.

Acknowledgements. This research has made use of SAXDAS linearized and cleaned event files produced at the BeppoSAX Science Data Center. We would like to thank the BeppoSAX SDC for the important assistance and the Mission Planning team for their fundamental contribution in performing the observations discussed in this work. We would also thank J. Stephen for a careful reading of the manustript. This research has been partially supported by ASI contracts $\mathrm{I} / \mathrm{R} / 103 / 00$ and $\mathrm{I} / \mathrm{R} / 107 / 00$. CV also acknowledges the financial support by Chandra X-ray Center grant DD1-2012X and by NASA LTSA grant NAG5-8107.

\section{References}

Bassani, L., Cappi, M., \& Malaguti, G. 1999, Astr. Lett. Comm., 39, 41

Chatzichristou, E. T. 2000, ApJ, 544, 712

Costantini, E., Nicastro, F., Fruscione, A., et al. 2000, ApJ, 544, 283

Dalgarno, A., \& Layzer, D. 1987, Spettroscopy of Astrophysical Plasmas (Cambridge Univ. Press., Cambridge) de Grijp, M. H. K., Keel, W. C., Miley, G. K., Goudfrooij, P., \& Lub, J. 1992, A\&AS, 96, 389

De Rosa, A., Fabian, A. C., Piro, L., \& Ballantyne, D. R. 2001, in the Proc. of the Symp. on New Visions of the X-ray Universe in the XMM-Newton and Chandra Era, ESTEC, The Netherlands

De Rosa, A., Piro, L., Fiore, F., et al. 2002, A\&A, 387, 838

Done, C., Mulchaey, J. S., Mushotzky, R. F., \& Arnaud, K. A. 1992, ApJ, 395, 275

Fiore, F., Guainazzi, M., \& Grandi, P. 1998, BeppoSAX Cookbook

Frontera, F., Costa, E., Dal Fiume, D., et al. 1997, A\&AS, 122, 357

Giommi, P., Tagliaferri, G., Beuermann, K., et al. 1991, ApJ, 378, 77

Kinney, A. L., Schmitt, H. R., Clarke, C. J., et al. 2000, ApJ, 537, 152

Landi, R., Bassani, L., Malaguti, G., et al. 2001, A\&A, 379, 46

Magdziarz, P., \& Zdziarski, A. 1995, MNRAS, 273, 837

Makishima, K. 1985, in ed. K. O. Mason, M. G. Watson, \& N. E. White, The Physics of Accretion onto Compact Objects (SpringerVerlag, Berlin), 249

Malizia, A., Bassani, L., Zhang, S. N., et al. 1999, ApJ, 519, 637

Marshall, F. E., Boldt, E. A., Holt, S. S., et al. 1979, ApJS, 40, 657

McNaron-Brown, K., Johnson, W. N., Jung, G. V., et al. 1995, ApJ, 451,575

Mineo, T., Fiore, F., Laor, A., et al. 2000, A\&A, 359, 471

Nandra, K., George, I. M., Mushotzky, R. F., Turner, T. J., \& Yaqoob, T. 1999, ApJ, 523, L17

Pellegrini, S., Cappi, M., Bassani, L., et al. 2000, A\&A, 353, 447

Piccinotti, G., Mushotzky, R. F., Boldt, E. A., et al. 1982, ApJ, 253, 485

Ruszkowski, M., \& Fabian, A. C. 2000, MNRAS, 315, 223

Schartel, N., Schmidt, M., Fink, H. H., Hasinger, G., \& Trumper, J. 1997, A\&A, 495, 749

Schmitt, H. R., \& Kinney, A. L. 2001, ApJS, 128, 479

Zdziarski, A. A., Poutanen, J., \& Johnson, W. N. 2000, ApJ, 542, 703

Zdziarski, A. A., Leighly, K. M., Matsuoka, M., Cappi, M., \& Mihara, T. 2002, ApJ, 573, 505 